\title{
Buccal Midazolam Solution for the Management of Prolonged Acute Convulsive Seizures: A Cost Analysis
}

\author{
Catherine Ludwig $^{1}$ (i) $\cdot$ Lauri Fisher $^{1}$
}

Published online: 11 July 2019

(c) The Author(s) 2019

\begin{abstract}
Background There is little published evidence on how pack size impacts on health system costs. In the UK, children and adolescents aged 10 to $<18$ years with prolonged acute convulsive seizures (PACS) occurring in the community setting are usually managed by the administration of buccal midazolam: Epistatus ${ }^{\circledR}$ or Buccolam ${ }^{\circledR}$. These two preparations have markedly differing cost structures, being sold at different prices and presented as single units versus 4-packs, respectively. Consequently, the cost-per-PACS and overall budget impact of the two products cannot be simply inferred but is instead dependent on the likelihood of use. Also relevant to its likelihood of use is midazolam's status as a controlled substance under the Misuse of Drugs Act.

Objective and Methods The purpose of this economic analysis was to present the cost implications of single-unit versus multi-pack prescribing of a rescue medication based on likelihood of use. There is little published evidence to inform the likelihood of a midazolam dose being used once prescribed. A Delphi survey was conducted with physicians $(n=5)$, nurses $(n=11)$ and pharmacists $(n=24)$ from the community and hospital healthcare settings in the UK to explore the frequency distribution of prolonged seizures in the general epileptic population per 6-month period, along with common patterns of prescribing. This informed a model of the budgetary impact of single-unit (Epistatus ${ }^{\circledR}$ ) versus 4-pack (Buccolam ${ }^{\circledR}$ ) prescribing. Results Respondents cited both 'wastage' and 'dispensing more than is likely to be used of a controlled drug' as poor clinical practice, which suggests that prescribing in multiples of four should be reserved for patients who are likely to experience PACS at a higher frequency. The Delphi survey and subsequent regression analysis found that PACS frequency distribution follows an exponential drop, with over half (55\%) of patients experiencing zero to one prolonged seizure(s) per 6-month period. Despite this, the majority (70\%) of low PACS frequency patients are prescribed buccal midazolam in multiples of four by their treating physician. When looking at overall budgetary impact, the strategy of prescribing single-unit Epistatus ${ }^{\circledR}$ versus 4-pack Buccolam ${ }^{\circledR}$ is cost saving in low PACS frequency patients who do not require multiple units (referred to as spare loading), at $-£ 15.33$ per patient per 6 months. If spare loading is universally applied to all patients irrespective of seizure frequency, the mean incremental cost of single unit Epistatus ${ }^{\circledR}$ instead of 4-pack Buccolam ${ }^{\circledR}$ equates to $£ 51.23$ per patient per 6 months. If spare loading is reserved for higher PACS frequency patients (two or more per 6 months), the universal prescribing of single-unit Epistatus ${ }^{\circledR}$ versus 4-pack Buccolam ${ }^{\circledR}$ would result in a mean incremental cost of $£ 17.82$ per patient per 6 months.

Conclusions The availability of single-unit Epistatus ${ }^{\circledR}$ allows for greater flexibility in prescribing. Physicians should attempt to categorise patients according to past PACS frequency with low PACS patients being prescribed single unit Epistatus ${ }^{\circledR}$ (with/without spare loading) and 4-pack Buccolam ${ }^{\circledR}$ reserved for patients in whom a high volume of usage is anticipated.
\end{abstract}

Electronic supplementary material The online version of this article (https://doi.org/10.1007/s41669-019-0164-x) contains supplementary material, which is available to authorized users.

Catherine Ludwig

catherine@mymedicaldepartment.com

1 My Medical Department Pty Ltd, 82 San Fernando Drive, Worongary, Australia 


\section{Key Points for Decision Makers}

Over half of patients are expected to experience zero to one prolonged acute convulsive seizure(s) per 6-month period. For these patients, single-unit Epistatus ${ }^{\circledR}$ remains a cheaper prescribing strategy than 4-pack Buccolam ${ }^{\circledR}$ $(-£ 15.33$ per patient per 6 months).

Universal use of Epistatus ${ }^{\circledR}$ for all patients would result in a mean incremental cost of $£ 51.23$ per patient per 6 months. Therefore, 4-pack Buccolam ${ }^{\circledR}$ should be prescribed for patients in whom a high volume of usage is anticipated.

\section{Introduction}

Epilepsy is the most common neurological disorder of adolescence $[1,2]$. It is a chronic neurological condition that affects approximately 1 in 103 people in the UK [3]. There are many different forms of epilepsy, all of which are characterised by recurring seizures that result from abnormal electrical activity in the brain [4]. Seizures may occur on their own or may be accompanied by other symptoms as part of a complex medical syndrome [2]. While the mainstay of treatment is the prevention of seizures through anti-epileptic drugs (AEDs), an estimated one-third of children and adolescents with epilepsy continue to experience breakthrough seizures despite optimal AED treatment [3]. A seizure becomes more difficult to terminate the longer it continues, and seizures lasting $>5 \mathrm{~min}$, also known as prolonged acute convulsive seizures (PACS), are likely to progress into convulsive status epilepticus if left untreated [5-8]. Status epilepticus is associated with increasing morbidity and mortality for the patient. Achieving early termination of PACS is therefore of paramount importance [5].

In the UK community setting, PACS were historically managed by the rectal administration of diazepam; however, the rectal route has now largely fallen out of favour due to practical and social concerns. Most guidelines today favour use of the buccal route, which is seen as quicker and easier to access, and avoids the social stigma of rectal administration $[9,10]$.

For children and adolescents aged 10 to $<18$ years, two buccal presentations of midazolam are now licensed in the UK: Epistatus ${ }^{\circledR}$ (Veriton Pharma, Weybridge, Surrey, UK) $10 \mathrm{mg} / 1 \mathrm{~mL}$ prefilled syringes (PFS) and Buccolam ${ }^{\circledR}$ (Shire Services BVBA, Brussels, Belgium) $10 \mathrm{mg} / 2 \mathrm{~mL}$ PFS [11, 12].
These two presentations have markedly different cost structures: Buccolam ${ }^{\circledR}$ PFS are sold in a 4-pack (list price ${ }^{1}$ of $10 \mathrm{mg} / 2 \mathrm{~mL}$ syringes $£ 91.50$ excluding value-added tax [VAT] for four doses) [13], whereas Epistatus ${ }^{\circledR}$ PFS is sold in single units (list price ${ }^{1}$ of $£ 45.76$ excluding VAT each) [14]. Consequently, the overall budget impact of the two products cannot be simply inferred but is instead dependent on the likelihood of the patient experiencing a PACS.

In addition to overall budget impact, a factor that should influence quantity prescribed is midazolam's status as a controlled substance under the Misuse of Drugs Act [15]. In 2010, there were an estimated 186,000 new benzodiazepine abusers and emergency departments saw a $139 \%$ increase in benzodiazepine-related visits in the USA [16]. High rates of midazolam injection among drug users in Bangkok, Thailand were also reported, calling for evidence-based approaches for reducing harm [17].

While the status of midazolam as a controlled substance is widely established, there is a paucity of information describing the frequency distribution of PACS in the general epileptic population, the prescribing habits of the clinicians or what happens to the midazolam once it is dispensed into the community. There is also little published evidence on how pack size impacts on health system costs. In order to inform a model of the budgetary impact of single-unit Epistatus ${ }^{\circledR}$ versus 4-pack Buccolam ${ }^{\circledR}$, a Delphi survey was conducted.

The Delphi technique, first described by Dalkey and Helmer in 1963, is a widely accepted method for achieving convergence of opinion concerning real-world knowledge $[18,19]$. In this technique, the panel is presented with multiple iterations of a questionnaire. Critically, between each round, the participants are presented with a summary of the replies of the whole group along with their own position on each question, before being presented with the next iteration of the survey [18-20]. In contrast to other informationgathering techniques, this gives the expert respondents an opportunity to re-assess their initial judgement in light of the answers provided by other members of the group, resulting in the convergence of opinion, and hence development of a robust group consensus [18-20]. The purpose of this analysis was to present the cost implications of single-unit versus multi-pack prescribing based on likelihood of use.

\footnotetext{
${ }^{1}$ Prices correct at the time of submission of the manuscript.
} 


\section{Methods}

\subsection{Prescribing and Dispensing of Buccal Midazolam}

In the absence of evidence to inform the likelihood of midazolam use once prescribed, a Delphi survey was conducted in April 2017. The survey was undertaken with a panel comprising physicians, nurses and pharmacists from community and hospital healthcare settings who work closely alongside patients with epilepsy and their carers (Table 1).

The healthcare professionals were selected from across Scotland, England and Wales using the Binley's UK database of medical professionals.

The objectives of the survey were as follows:

1. Explore the rate of PACS (number per 6 months) in the general epilepsy population.

2. Quantify the practical aspects of prescribing and dispensing buccal midazolam for PACS in routine clinical practice.

3. Understand how use is tracked and ascertain likelihood of misuse or diversion and potential wastage in the community.

4. Explore how the availability of a PFS sold in individual units might impact on prescribing patterns.

The Delphi process involved two rounds. The first round consisted of a questionnaire, with initial results being presented back to all participants. This provided an opportunity for comment or revision to obtain a consensus and determine the local pattern of care. This was followed by a second round of the questionnaire.

Table 1 Composition of the Delphi panel

\begin{tabular}{llcc}
\hline Speciality & Location & Number & Total \\
\hline Physician & Tertiary & 1 & 5 \\
& Secondary & 3 & \\
& Primary & 1 & \\
Nurses & Hospital & 5 & 11 \\
& Community & 6 & \\
Pharmacists & Hospital & 19 & $24^{\mathrm{a}}$ \\
& Community & 2 & \\
Final respondents & Other & 3 & 36 \\
\hline
\end{tabular}

${ }^{a}$ Four pharmacists dropped out after Round 1

\subsection{Clinically Established Assumptions}

Certain clinically established assumptions were used to inform the economic model. In accordance with the label of both products, only one dose is assumed to be administered per treatment episode. The Delphi survey and results derived from it apply to a typical patient population (as in a general epilepsy clinic). The two formulations were presumed equivalent in safety and efficacy, and consequently no difference in seizure termination rates or healthcare resource utilisation costs have been included in the model.

\subsection{Pooled Analysis}

The original study segmented data collection and analysis based on age: $\geq 10$ to $<13, \geq 13$ to $<16$ and $\geq 16$ to $<18$ years. Due to homogeneity, a pooled analysis was completed for ease of reporting.

Physician, nurse and pharmacist data were combined across all areas of analysis: prolonged seizure frequency, current prescribed treatment option for specific patient types, quantity of rescue medication required, wastage and divergence, and extent to which availability of a single unit would change prescribing.

\subsection{List Price}

In 2019 the list price was $£ 45.76$ for Epistatus ${ }^{\circledR} 10 \mathrm{mg} / 1 \mathrm{~mL}$ (single PFS unit) and $£ 91.50$ for Buccolam ${ }^{\circledR} 10 \mathrm{mg} / 2 \mathrm{~mL}$ (4-pack PFS) $[13,14]$.

\subsection{Economic Model}

Regression analysis was used to explore the relationship between the rate of PACS and percentage of patients experiencing each rate, i.e., the PACS frequency distribution, where $x$ is the PACS frequency (number of PACS per 6 months) and $y$ is the proportion (\%) of patients who have PACS frequency $x$.

The number of packs of single-unit Epistatus ${ }^{\circledR}$ or 4-pack Buccolam ${ }^{\circledR}$ required for each PACS frequency $(x)$ was also described mathematically (Table 2 ) according to the different prescribing habits elucidated by the survey, namely spare loading versus no spare loading. This was multiplied

Table 2 Quantity of packs dispensed

\begin{tabular}{lll}
\hline Loading & $\begin{array}{l}\text { Epistatus }^{\circledR} \text { single } \\
\text { unit }\end{array}$ & Buccolam $^{\circledR}$ 4-pack \\
\hline No spare loading & $(x+1)$ & $\left\lfloor\frac{x}{4}\right\rfloor+1$ \\
With spare loading & $(x+2)$ & $\left\lfloor\frac{x+1}{4}\right\rfloor+1$
\end{tabular}

$x$ frequency of seizures per 6 months 
Table 3 Cost of medication

\begin{tabular}{lll}
\hline Loading & Epistatus $^{\circledR}$ single unit & Buccolam $^{\circledR}$ 4-pack \\
\hline No spare loading & $\left(L_{\mathrm{E}}\right) \times(x+1) \times y$ & $\left(L_{\mathrm{B}}\right) \times\left(\left\lfloor\frac{x}{4}\right\rfloor+1\right) \times y$ \\
With spare loading & $\left(L_{\mathrm{E}}\right) \times(x+2) \times y$ & $\left(L_{\mathrm{B}}\right) \times\left(\left\lfloor\frac{x+1}{4}\right\rfloor+1\right) \times y$
\end{tabular}

$L_{B}$ list price of Buccolam ${ }^{\circledR}, L_{E}$ list price Epistatus ${ }^{\circledR}, x$ frequency of seizures per 6 months, $y$ proportion (\%) of patients who have prolonged acute convulsive seizures frequency $x$

Table 4 Difference in cost of medication

\begin{tabular}{ll}
\hline Loading & Difference in cost $\left(\right.$ Epistatus $^{\circledR}-$ Buccolam $\left.^{\circledR}\right)$ \\
\hline No spare loading & {$\left[L_{\mathrm{E}}(x+1)-L_{\mathrm{B}}\left(\left\lfloor\frac{x}{4}\right\rfloor+1\right)\right] \times y$} \\
With spare loading & {$\left[L_{\mathrm{E}}(x+2)-L_{\mathrm{B}}\left(\left\lfloor\frac{x+1}{4}\right\rfloor+1\right)\right] \times \mathrm{y}$} \\
\hline
\end{tabular}

$L_{B}$ list price of Buccolam ${ }^{\circledR}, L_{E}$ list price of Epistatus ${ }^{\circledR}, x$ frequency of seizures per 6 months, $y$ proportion (\%) of patients who have prolonged acute convulsive seizures frequency $x$

by the product list price (L) to give an estimate of costper-6 months. Finally, the cost-per- 6 months was multiplied by the percentage of patients experiencing each rate of PACS $(y)$ to give the overall budgetary impact of single-unit Epistatus $^{\circledR}$ versus 4-pack Buccolam ${ }^{\circledR}$, as shown in Table 3.

The difference in cost is obtained by simple subtraction (Epistatus $^{\circledR}-$ Buccolam $^{\circledR}$ ), as outlined in Table 4.

\section{Results}

\subsection{Clinical Behaviours}

The key results from the Delphi survey are outlined in the Electronic Supplementary Material. Of particular importance were the behaviours and views around prescribing and dispensing buccal midazolam as an emergency rescue medication:

- Clinicians use recent PACS history to prescribe against future risk.

- Spare loading was universally considered clinically necessary in higher PACS frequency patients, meaning prescribers prefer their patients to be dispensed at least one 'extra' syringe in addition to the anticipated requirement. This is in order to ensure the patient is never left without a dose immediately after experiencing a PACS.

- The panel considered 55\% of patients to be 'low frequency', defined as experiencing zero to one PS per 6-month period.
- Despite classification as low frequency, physicians prescribed Buccolam ${ }^{\circledR}$ as a 4-pack to $70 \%$ of these patients.

- $75 \%$ of respondents considered wastage to be an issue with 4-pack Buccolam ${ }^{\circledR}$. This was seen as an especial concern given the narcotic nature of midazolam.

- The risk of diversion of surplus dispensed medication was reported to be of particular concern in the adolescent patient group.

- Although pharmacists are legally entitled to split 4-pack Buccolam ${ }^{\circledR}$ in order to fill a prescription for fewer than four Buccolam ${ }^{\circledR}$ PFS, $50 \%$ of respondents were unaware of this.

\subsection{Rate of Prolonged Acute Convulsive Seizures (PACS)}

The PACS frequency data were found to fit an exponential curve, modelled by the function $y=0.3353 \mathrm{e}^{-0.435 x}$ with a high degree of accuracy $\left(R^{2}>0.99\right.$; Fig. 1$)$, where $x$ is the PACS frequency (number of PACS per 6 months) and $y$ is the proportion (\%) of patients who have PACS frequency $x$.

\subsection{Cost Per PACS Without and With Spare Loading}

Based on the dominant reported prescribing patterns of 'spare loading' versus 'no spare loading', the quantity of packs dispensed per 6-month time horizon is summarised in Table 2. The associated drug cost is illustrated graphically in Fig. 2 .

Importantly, the required quantity of Epistatus ${ }^{\circledR}$ increases linearly with the number of PACS $(x)$, whereas the required quantity of Buccolam ${ }^{\circledR}$ increases in a stepwise manner due to it being supplied in quantities of four. Consequently, the cost relationship between the two agents varies markedly according to two key factors:

- the number of PACS $(x)$; and

- whether or not the prescriber uses spare loading.

Our formula $y=0.3353 \mathrm{e}^{-0.435 x}$ predicts that $33.5 \%$ of patients will remain free of PACS over a 6-month period. In these patients, the cost of prescribing single-unit Epistatus ${ }^{\circledR}$ is $£ 45.76$ versus $£ 91.50$ for 4 -pack Buccolam ${ }^{\circledR}$. If spare loading is desired by the prescriber, an additional single-unit Epistatus ${ }^{\circledR}$ will cost $£ 45.76$, totalling $£ 91.52$ per patient per 6 months (incremental cost of $£ 0.02$ vs. 4 -pack Buccolam ${ }^{\circledR}$ ).

\subsection{Budget Impact}

The difference in cost of medication is presented in Table 4 and Fig. 2. This was multiplied by the PACS frequency distribution function $y=0.3353 \mathrm{e}^{-0.435 x}$ to give the budget impact per 100 patients, cumulatively across all values of $x$ $(0 \rightarrow \infty)$. This is presented graphically in Fig. 3 . 
Fig. 1 Prolonged acute convulsive seizures frequency distribution per 6 months
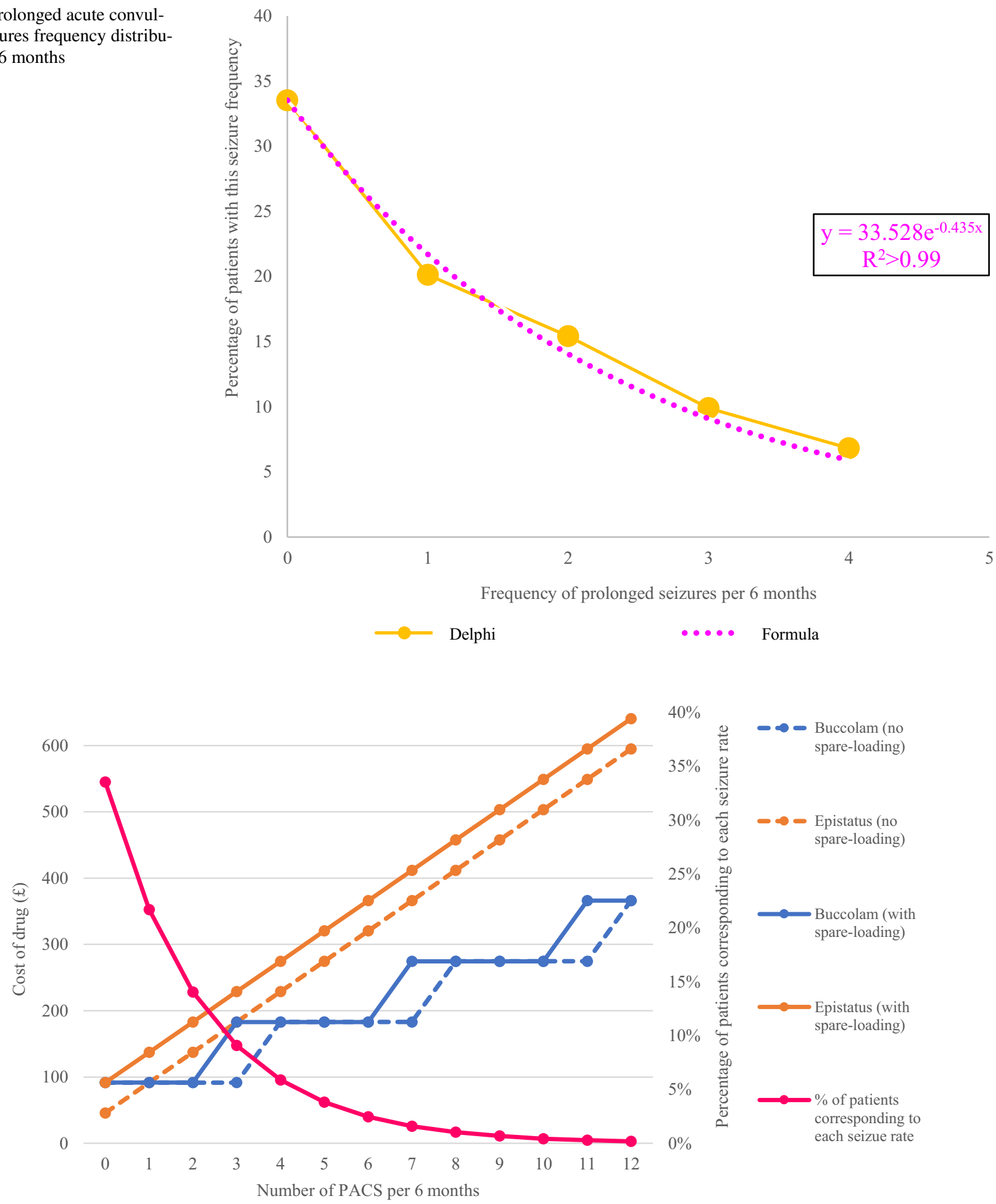

Fig. 2 Prolonged acute convulsive seizures (PACS) rate per 6 months and associated drug cost 


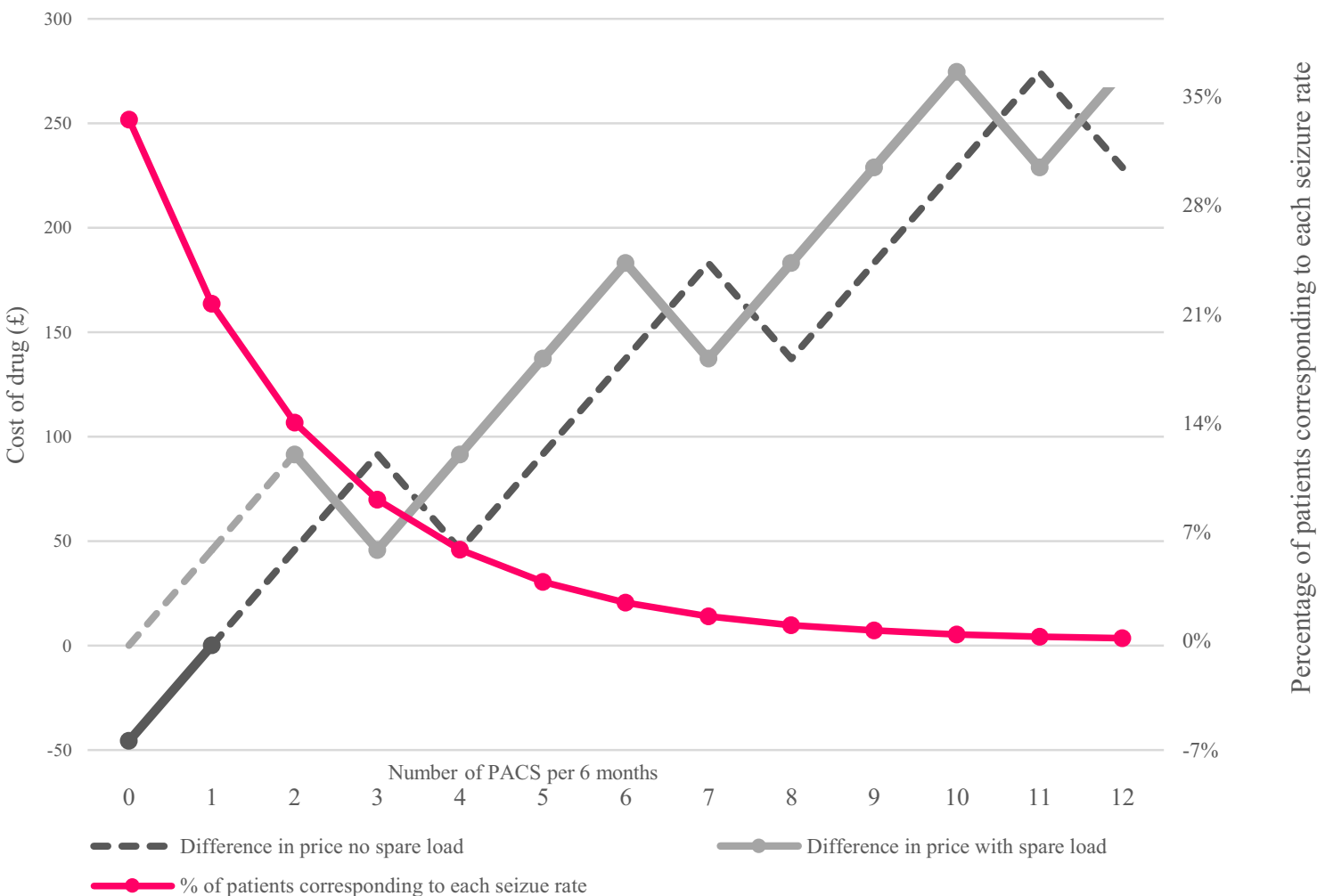

Fig. 3 Prolonged acute convulsive seizures (PACS) rate per 6 months and difference in cost with and without spare loading

Table 5 Budget impact of universally prescribing singleunit Epistatus ${ }^{\circledR}$ versus 4-pack Buccolam $^{\circledR}$

\begin{tabular}{|c|c|c|}
\hline PACS Frequency & No spare load (£) & With spare load (£) \\
\hline Low PACS frequency ( $0-1$ per 6 months) & $-1533.13(-15.33)$ & $994.16(9.94)$ \\
\hline Any seizure frequency integral $0 \rightarrow \infty$ & $1782.19(17.82)$ & $5122.72(51.23)$ \\
\hline $\begin{array}{l}\text { Spare load reserved for higher frequency }(\geq 2 \\
\text { PACS per } 6 \text { months) }\end{array}$ & 25.95 per patient per 6 months & \\
\hline
\end{tabular}

Data given as total incremental $£$ per 100 patients per 6 months (average cost for one patient, $£$ per 6 months), unless otherwise stated
For patients who carry one dose and replace it as needed ('no spare loading'):

- In the almost $34 \%$ of patients who will not experience another PACS within 6 months, single-unit Epistatus ${ }^{\circledR}$ is $£ 44.74$ cheaper than 4-pack Buccolam ${ }^{\circledR}$ and is cost neutral $(+£ 0.02)$ in an additional $21.7 \%$ of patients who will only experience one PACS per 6 months. Therefore, cumulatively, in over half of consultations (55.2\% of epilepsy patients who experience PACS), singleunit Epistatus ${ }^{\circledR}$ is cost saving or neutral versus 4-pack Buccolam ${ }^{\circledR}$.
Table 5 shows the budget impact of universal prescribing:

- Universal use of single-unit Epistatus ${ }^{\circledR}$ for all patients instead of 4-pack Buccolam ${ }^{\circledR}, x(0 \rightarrow \infty)$, would result in a mean incremental cost of $£ 17.82$ per patient per 6 months.

For patients who carry an additional dose to always have one spare ('with spare loading'):

- Single-unit Epistatus ${ }^{\circledR}$ is cost neutral for seizure-free patients $(+£ 0.02)$ but is more expensive than 4-pack Buccolam ${ }^{\circledR}$ for all other values of $x$. 
- Universal use of single-unit Epistatus ${ }^{\circledR}$ for all patients instead of 4-pack Buccolam ${ }^{\circledR}$ would result in a mean incremental cost of $£ 51.23$ per patient per 6 months.

If spare loading were reserved only for non-low PACS frequency patients (two or more PACS per 6 months), the budget implications of this approach would be as follows:

- Prescribing single-unit Epistatus ${ }^{\circledR}$ with universal application of this prescribing strategy would result in a mean incremental cost of $£ 25.95$ per patient per 6 months versus 4-pack Buccolam ${ }^{\circledR}$.

\section{Discussion}

In the UK, buccal midazolam $10 \mathrm{mg}$ for use in the community is given to children and adolescents aged 10 to $<18$ years who have had a previous episode of prolonged or serial convulsive seizures [10]. Our comparative cost analysis suggests that treatment with single-unit Epistatus ${ }^{\circledR}$ is cost saving compared with 4-pack Buccolam ${ }^{\circledR}$ in $34 \%$ of consultations and cost neutral in $21.7 \%$ of consultations. These savings are attributable to non-uniform PACS frequency distribution and product presentation as a single unit versus a 4-pack.

A Delphi survey was conducted to inform PACS frequency. This technique is particularly well-suited to new research areas and exploratory studies as its approach is used to obtain the most reliable consensus from a panel of experts [18-20]. Feedback of individual contributions allows the opportunity to revise views, while avoiding direct confrontation of the experts [20]. In this way, equal weight is given to all those who participate and the risk of an individual or group of individuals being overly influential or dominant in the process is reduced [21, 22]. Used correctly and rigorously, the Delphi technique can contribute significantly to broadening knowledge [20,22]. While it can be criticised that the existence of a consensus does not mean that the correct answer has been found, the collective judgment of experts, although made up of subjective opinions, is considered to be more reliable than individual statements and is thus more objective in its outcomes [21,22].

In order to interpret the economic findings within the context of everyday clinical practice, a number of additional parameters regarding treatment of PACS were investigated. Behaviours around prescribing and dispensing buccal midazolam as an emergency rescue medication were of particular interest.

When considering low-frequency PACS patients (zero to one per 6 months), the generated data indicated overprescribing in this patient group. When participants were asked if they considered wastage to be an issue with 4-pack Buccolam ${ }^{\circledR}$, most respondents answered yes and considered the potential for diversion to be of particular concern amongst teenagers.

This is supported by midazolam's status as a controlled substance under the misuse of drugs legislation [15]. Benzodiazepine misuse and abuse is a growing problem. The number of admissions to treatment programmes for benzodiazepine abuse nearly tripled from 1998 to 2008 [16]. It has been suggested that midazolam injection, partly because of the associated amnestic effects, can result in elevated rates of risk behaviour, including syringe sharing, and that withdrawal effects, including headaches, insomnia and agitation, are typical of benzodiazepines and can be fatal [17].

Nonetheless, as with other rescue medications such as adrenaline autoinjectors, a preference for 'spare loading' was reported by the majority of respondents. This allows for rescue medication to be kept in multiple locations, i.e., school and home. For this reason, our cost-effectiveness assessment was extended to take this clinical practice into account. However, spare loading may not be clinically necessary for low PACS frequency patients. Theoretically, they could be managed by carrying the device and issuing a repeat prescription, to be dispensed only if the initial midazolam dose is used. Prescribing in this way may have financial benefits for the healthcare system.

Given it is estimated that as much as $£ 300$ million is wasted every year in the UK on unused or partially used medication and $£ 150$ million of this waste is avoidable [23], it seems reasonable to reserve 4-pack prescribing for highusage patients now that a single-unit Epistatus ${ }^{\circledR}$ is available.

To test this assumption, physicians were asked if availability of single-unit Epistatus ${ }^{\circledR}$ would change prescribing for low PACS frequency patients aged 10 to $<18$ years, to which $100 \%$ of the sample indicated it would.

This survey supports the view that a universal strategy for prescribing is inappropriate. Instead, the patient's condition should be assessed and the quantity of rescue medication prescribed accordingly. Our recommendation is that prescribing in multiples of four should not be initiated in low PACS frequency patients, but instead should only be recommended for patients in whom a high volume of usage is anticipated.

The model is based on UK costs and healthcare professional treatment decisions. However, the results are likely to be relevant to countries with similar healthcare systems.

Previous studies have evaluated the cost effectiveness of buccal midazolam against rectal diazepam [24-27]. These studies have shown greater cost savings with buccal midazolam than with rectal diazepam despite greater drug acquisition costs [24-27]. However, this is the first published analysis to look at two different presentations of buccal midazolam and to take into consideration PACS frequency. 
To our knowledge, this is also the first published analysis of the budget impact of single-unit versus multi-pack prescribing of a rescue medication, taking into consideration the likelihood of its being needed.

The study has several limitations, notably the following:

1. The time horizon of 6 months is shorter than the exfactory shelf-life of both products. However, the study revealed that clinicians use recent seizure history to inform prescribing against future risk. This supports the extrapolation of the data to longer time periods.

2. The estimated risk distribution for seizures is entirely based on expert opinion rather than epidemiological data.

3. No adjustment has been made for the robustness or durability of the Epistatus ${ }^{\circledR}$ versus Buccolam ${ }^{\circledR}$ devices after opening their primary packaging. However, in the absence of head-to-head data supporting this claim, the presence of a protective secondary packaging around each individual Epistatus ${ }^{\circledR}$ device suggests that any such adjustment would possibly have influenced the results in its favour. Regardless, no adjustment has been made for this.

4. Finally, no adjustment has been made for differences in shelf-life ex-factory, as there is no guarantee there will be a difference in product longevity at the time it is dispensed in the pharmacy.

\section{Conclusion}

With approximately $33 \%$ of PACS patients not experiencing an episode within a 6-month period, prescribing single-unit Epistatus ${ }^{\circledR}$ would be cost saving (no spare loading) or cost neutral (with spare loading) while reducing drug wastage and the potential risk of drug diversion. As it is difficult to predict which patients will remain PACS free, the methodology in this paper was developed to compare the costs of a multi-pack versus single-unit product in a chronic disease that requires sporadic treatment of acute episodes. The analysis showed that in low PACS frequency patients, who represent $55.2 \%$ of the patient population, the universal use of single-unit Epistatus ${ }^{\circledR}$ instead of 4-pack Buccolam ${ }^{\circledR}$ would result in a mean cost saving of $£ 15.33$ per patient. If single-unit Epistatus ${ }^{\circledR}$ with spare loading is prescribed universally to all patients irrespective of seizure frequency, there is an incremental increase in cost of $£ 51.23$ per patient per 6 months compared with universal prescribing of 4-pack Buccolam ${ }^{\circledR}$. The availability of single-unit Epistatus ${ }^{\circledR}$ allows for greater flexibility in prescribing. Physicians should attempt to categorise patients according to past PACS frequency, with low PACS patients being prescribed single-unit Epistatus ${ }^{\circledR}$ (with/ without spare loading) and 4-pack Buccolam ${ }^{\circledR}$ reserved for patients in whom a high volume of usage is anticipated.

Author Contributions The study was designed by InTeg Health with input from Catherine Ludwig. The methods were implemented by InTeg Health. The mathematical models were devised by CL. CL and LF participated in the review and interpretation of the data analysis. The manuscript was written by CL and LF. CL is the guarantor for the overall content of this paper.

Data Availability Statement The datasets generated and/or analysed during the current study are available from the corresponding author on reasonable request.

\section{Compliance with Ethical Standards}

Funding This study was funded by Veriton Pharma, the manufacturer of Epistatus ${ }^{\circledR}$ (midazolam buccal solution). My Medical Department Pty Ltd. provides medical consultancy for Veriton Pharma. The authors conducted all analyses and wrote the manuscript. The decision to submit the manuscript for publication was made by the authors.

Conflict of Interest Catherine Ludwig is the director of My Medical Department. Lauri Fisher is a Senior Scientific Advisor at My Medical Department.

Open Access This article is distributed under the terms of the Creative Commons Attribution-NonCommercial 4.0 International License (http://creativecommons.org/licenses/by-nc/4.0/), which permits any noncommercial use, distribution, and reproduction in any medium, provided you give appropriate credit to the original author(s) and the source, provide a link to the Creative Commons license, and indicate if changes were made.

\section{References}

1. Appleton RE, Neville GRN. Teenagers with epilepsy. Arch Dis Child. 1999;81(1):76-9.

2. Wheless JW, Kim HL. Adolescent seizures and epilepsy syndromes. Epilepsia. 2002;43(Suppl 3):33-5.

3. Joint Epilepsy Council of the UK and Ireland. Epilepsy prevalence, incidence and other statistics. http://www.epilepsyscotlan d.org.uk/pdf/Joint_Epilepsy_Council_Prevalence_and_Incid ence_September_11_(3).pdf. Accessed 2011.

4. Goldenberg MM. Overview of drugs used for epilepsy and seizures: etiology, diagnosis, and treatment. Pharm Ther. 2010;35(7):392-415.

5. Laxer KD, Trinka E, Hirsch LJ, Cendes F, Langfitt J, Delanty N, et al. The consequences of refractory epilepsy and its treatment. Epilepsy Behav. 2014;37:59-70.

6. Divino V, Petrilla AA, Bollu V, Velez F, Ettinger A, Makin C. Clinical and economic burden of breakthrough seizures. Epilepsy Behav. 2015;51:40-7.

7. Epilepsy Foundation. Status epilepticus. 2014. https://www.epile psy.com/learn/challenges-epilepsy/seizure-emergencies/statu s-epilepticus. Accessed 6 Dec 2018

8. Epilepsy Action. Treatment and care for seizures that last more than 5 minutes and for status epilepticus. 2015. https://www.epile 
psy.org.uk/info/firstaid/emergency-treatment-seizures-last-longtime. Accessed 6 Dec 2018

9. Glauser T, Shinnar S, Gloss D, Alldredge B, Arya R, Bainbridge $\mathrm{J}$, et al. Evidence-based guideline: treatment of convulsive status epilepticus in children and adults: report of the Guideline Committee of the American Epilepsy Society. Epilepsy Curr. 2016;16(1):48-61.

10. National Institute for Health and Clinical Excellence. The epilepsies: the diagnosis and management of the epilepsies in adults and children in primary and secondary care. NICE clinical guideline CG137. London: NICE; 2012.

11. Medicines. Epistatus-summary of product characteristics (SPC)—(eMC). 2018. https://www.medicines.org.uk/emc/produ ct/2679/smpc. Accessed 6 Dec 2018

12. Medicines. Buccolam-summary of product characteristics (SPC)—(eMC). 2018. https://www.medicines.org.uk/emc/produ ct/7460/smpc. Accessed 6 Dec 2018

13. NHS Electronic Drug Tariff. Buccolam. 2018. http://www. drugtariff.nhsbsa.nhs.uk/\#/00667352-DB/DB00667347/Home. Accessed 6 Dec 2018

14. NHS Electronic Drug Tariff. Epistatus. 2018. http://www. drugtariff.nhsbsa.nhs.uk/\#/00667352-DB/DB00667347/Home. Accessed 6 Dec 2018

15. Cahal DA. Misuse of drugs act 1971. Br Med J. 1974;1(5897):70-2.

16. Schmitz A. Benzodiazepine use, misuse, and abuse: a review. Ment Health Clin. 2016;6(3):120-6.

17. Kerr T, Kiatying-Angsulee N, Fairbairn N, Hayashi K, Suwannawong P, Kaplan K, et al. High rates of midazolam injection among drug users in Bangkok, Thailand. Harm Reduct J. 2010;7:7.

18. Hsu C, Sandford BA. The Delphi technique: making sense of consensus. Pract Assess Res Eval. 2007;12(10):1531-7714.

19. Young SJ, Jamieson LM. Delivery methodology of the Delphi: a comparison of two approaches. J Park Recreat Adm. 2001;19(1):42-58.
20. Ludwig B. Predicting the future: have you considered using the Delphi methodology? J Ext. 1997;35(5):1-4.

21. Okoli C, Pawlowski S. The Delphi method as a research tool: an example, design considerations and applications. Inf Manag. 2004;42(1):15-29.

22. Keeney S, Hasson F, McKenna HP. A critical review of the Delphi technique as a research methodology for nursing. Int J Nurs Stud. 2001;38(2):195-200.

23. BMA Patient Liasion Group. Dispensed but unopened medications. 2013. https://www.bma.org.uk/collective-voice/committees /patient-liaison-group/resources/dispensed-but-unopened-medic ations. Accessed 6 Dec 2018

24. Lee D, Gladwell D, Batty AJ, Brereton N, Tate E. The cost effectiveness of licensed oromucosal midazolam $\left(\right.$ Buccolam $\left.^{\circledR}\right)$ for the treatment of children experiencing acute epileptic seizures: an approach when trial evidence is limited. Pediatr Drugs. 2013;15(2):151-62.

25. Lee DC, Gladwell D, Hatswell AJ, Porter J, Brereton N, Tate E, et al. A comparison of the cost-effectiveness of treatment of prolonged acute convulsive epileptic seizures in children across Europe. Health Econ Rev. 2014;12(4):6.

26. Raspall-Chaure M, Martinez-Bermejo A, Sanchez-Carpintero R, Ruiz-Falco Rojas ML, Verdu-Perez A, Smeyers-Dura P, et al. Cost-effectiveness of buccal midazolam in the treatment of prolonged convulsive seizures in the outpatient setting in Spain. Rev Neurol. 2014;58(11):481-6.

27. Beghi E, Capovilla G, Franzoni E, Minicucci F, Romeo A, Verrotti A, et al. Midazolam vs. diazepam in prolonged seizures in children: a pharmacoeconomic approach. Acta Neurol Scand. 2018;137(1):24-8. 